\title{
New record of Trichoglossum rasum from Asia
}

\author{
Prabhugaonkar $\mathbf{A}^{1}$ and Pratibha $\mathbf{J}^{\mathbf{2}}$ \\ ${ }^{1}$ Botanical Survey of India, Eastern Regional Centre, Shillong 79300, India \\ ${ }^{2}$ A4/G2, Vijaynagar Co-operative Housing Society, Corlim, Goa-403110. Email: jalmipratibha@ rediffmail.com
}

Prabhugaonkar A, Pratibha J. 2017 - New record of Trichoglossum rasum from Asia. Mycosphere 8(4), 583-591, Doi 10.5943/mycosphere/8/4/7

\begin{abstract}
Trichoglossum rasum, a rare species in the genus Trichoglossum, previously reported only from New Caledonia in 1909, was recently observed in Khasi hills, India. This paper illustrates important distributional record along with first molecular phylogenetic placement of the species. A review of all 19 hitherto accepted species in the genus is also provided.
\end{abstract}

Key words - Fungal diversity, Geoglossaceae, Khasi hills

\section{Introduction}

The Khasi hills in the Indian state of Meghalaya have a wide range of flora and fauna. The diverse vegetation is unique in having a mixture of Asiatic and Indian Peninsular elements. The Environment Report, Meghalaya, (2005) has expressed concerns that species that were common about 20 to 30 years ago have become rare due to anthropogenic pressures such as overexploitation, deforestation, mining, shifting cultivation and habitat destruction. In this regard, efforts are being made to document the biodiversity resources. In one of such effort, an ascomycetous fungus, commonly called 'earth tongue' and belonging to Geoglossaceae was collected. On microscopic examination, it was determined to belong to genus Trichoglossum Boud. (Boudier, 1885) with distinct trichomes on the surface and setae associated with Asci. The genus Trichoglossum has 19 accepted species (Table 1) till date (Index fungorum 2016). On careful morphological comparison, the species was identified as Trichoglossum rasum Pat. (Patouillard 1909) and thus forming a rare taxonomic record from the region. A first molecular phylogenetic analysis of species was performed to support this work.

\section{Materials \& Methods}

\section{Collection and culturing}

Freshly gathered samples from botanical garden of Botanical Survey of India, Eastern Regional Centre, Shillong, Meghalaya, were photo-documented and examined under a stereomicroscope. Fungal fruiting bodies were hand-sectioned, mounted in lactophenol and observed under a light microscope. Repeated attempts of culturing fungus failed. Silica gel dehydrated fruiting bodies (aerial tissue) were used for DNA isolation and sequencing. Sequencing was done at Rajiv Gandhi Centre for Biotechnology, Thiruvananthpuram, Kerala, India. The holotype is deposited in the herbarium of the Indian Agricultural Research Institute, New Delhi, 
India (HCIO) and isotype at herbarium of Botanical survey of India, Shillong (ASSAM). The taxonomic record has been deposited in 'Facesoffungi' database (Jayasiri et al. 2015).

\section{DNA isolation and PCR Analysis}

Silica gel dehydrated fruiting bodies (aerial tissue) were used for DNA isolation and sequencing. DNA isolation and PCR analysis was done according to Prabhugaonkar \& Bhat (2011). The 5.8S nuclear ribosomal gene with the two-flanking internal transcribed spacers (ITS), 28S nrDNA sequence (LSU) were amplified and sequenced using the primer pairs ITS-1F + ITS4R (White et al. 1990), LR5 + LROR (Crous et al. 2009) respectively. The sequence quality was checked using Sequence Scanner Software v1 (Applied Biosystems). Sequence alignment and required editing of the obtained sequences were carried out using Geneious Pro v5.1 (Drummond et al. 2010).

\section{Sequence alignment and phylogenetic analysis}

The sequences were blasted in GenBank with Blastn. ITS and LSU data sets generated based on the blasts and available literature (Hustad et. al. 2013). The combined data matrix was aligned using MEGA 7.0.18 to allow maximum alignment and maximum sequence similarity. A phylogenetic analysis was conducted using maximum likelihood (ML) in MEGA 7.0.18 (Kumar et al. 2008) with 1000 bootstrap replicates. The most suitable substitution models for the respective data sets were selected by using MEGA 7.0.18. Kimura 2-parameter model with Gamma distribution was used in analysis. Gaps were treated as a pair wise deletion and trees were viewed with MEGA 7.0.18. All newly generated sequences used in this study are deposited in GenBank.

\section{Results}

\section{Taxonomy}

Trichoglossum rasum Pat., Bull. Soc. mycol. Fr. 25: 130. 1909

Facesoffungi number: FoF02878

Saprobic in soil. Fruting body 3-4.5 cm, black, stipitate, erect, clavate to spatulate, with compressed ascogenous portion, ellipsoidal and inflated, with distinctly visible trichomes under hand lens; stipe 1.5-3.5 cm long, up to $3 \mathrm{~mm}$ thick. Trichomes dark brown, stiff. Hymenium with dark brown setae. Setae 200-250 × 5-12 $\mu \mathrm{m}$, septate, smooth, straight to flexuous, dark brown. Paraphyses 1.5-4.5 $\mu \mathrm{m}$ wide, filamentous, septate, bulbous and curved at tips. Asci 200-240 $\times 20$ $30 \mu \mathrm{m}$, unitunicate, consistently 8-spored, cylindrical to clavate, rounded at apex, short-pedicellate at base. Ascospores 110-140 × 5-7 $\mu \mathrm{m}$, mostly 7-septate, rarely 6-8-septate, straight or slightly curved, hyaline when young, dark brown when mature, narrowed and rounded at both ends, slightly wider in the middle portion, smooth.

Known distribution - Known from only collection in New Caledonia in 1909 and current collection in East Khasi hills, Meghalaya, India.

Material examined: India, Meghalaya, East Khasi hills District, Shillong, 4 July 2016, in garden soil amongst herbaceous vegetation, A. Prabhugaonkar, Herb. No. HCIO 52051; AVP-101 (ASSAM).

\section{Discussion}

The genus Trichoglossum Boud. was established in 1885 (Boudier, 1885) and is distinct from other genera in the family Geoglossaceae in having black projecting trichomes on surface of ascomata and setae amongst asci and paraphyses in transverse section. The genus is typified by Trichoglossum hirsutum (Pers.) Boud. 1907.

Trichoglossum rasum is a distinct species with asci up to $200 \mu \mathrm{m}$ long and ascospores 115 $140 \times 7-9 \mu \mathrm{m}$ and mostly 7-9-septate (Patouillard 1909). Our collection matches with the original description except that the asci are 200-240 $\mu \mathrm{m}$ (Patouillard 1909 has not given measurement 
range) and ascospores are 7 to rarely 8 -septate. We consider the new collection is the same species though they are geographically distant collections. This forms sixth species of Geoglossaceae reported from India with the other five being Geoglossum glabrum Pers. ex. Fr., G. alveolatum (Rehm.) Durand, Trichoglossum hirsutum (Pers. ex. Fr.) Bourd., T. velutipes (Peck) Durand (Agnihothrudu \& Barua 1962) and T. walteri (Das 2009). Nannfeldt 1942 considered T. wrightii similar to T. rasum without treating it as a synonym. T. wrightii is distributed in Bermuda (Durand, 1908), China (Teng, 1934), Cuba (type locality of T. hirsutum f. wrightii), Java (Rifai, 1965), Panama (Nannfeldt, 1942) and India: Uttar Pradesh, Mussoorie (Thind \& Singh 1965 as unidentified specimen). However, we consider T. wrightii as distinct species which is confirmed by its current treatment in Index fungorum/ MycoBank. T. wrightii is distinct from our collection with larger size of asci which is $240-265 \mu \mathrm{m}$ and variation in spore septation which is mostly 8-9septate, rarely 5-7-septate.

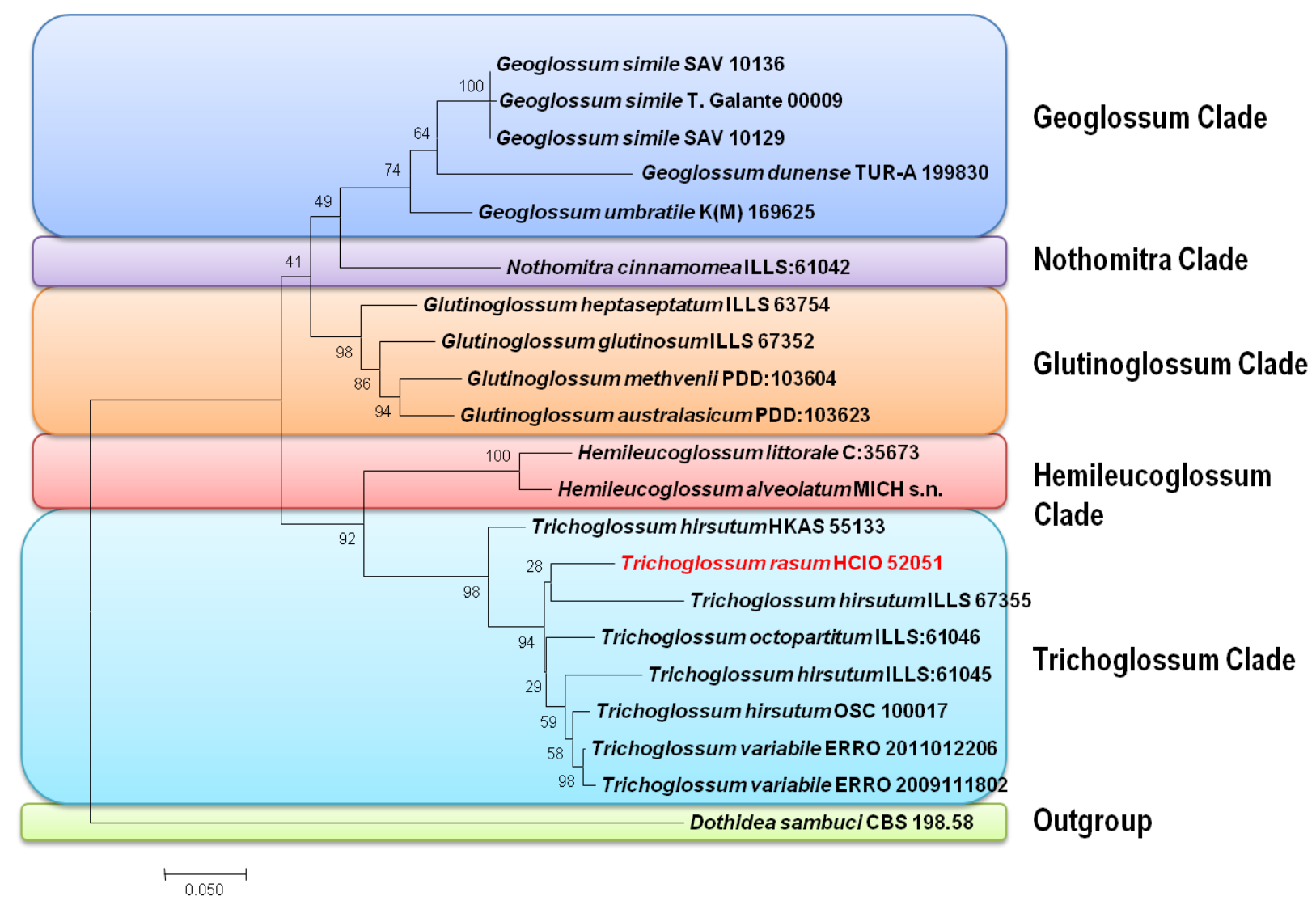

Figure 1 - Maximum likelihood (ML) tree inferred from ITS and LSU showing the relationship of Trichoglossum rasum with other species of Trichoglossum and related genera in family Geoglossaceae. Species sequenced and reported in the present paper is in red.

Molecular phylogeny confirms placement of the species in Trichoglossum. Only 4 out of 19 species described till date have molecular data. T. hirsutum, with good molecular data from collections around the world has been observed to be polyphyletic in nature (Hustad et. al. 2013). Current collection is morphologically distinct from $T$. hirsutum and same is confirmed by molecular phylogeny.

\section{Acknowledgements}

Authors are thankful to A.A. Mao, Head of office, BSI-ERC for support and suggestions on manuscript. The Director, Botanical Survey of India, Kolkata, for facilities. HCIO, Indian Agricultural Research Institute, New Delhi, India is acknowledged for deposition of material. 
Table 1 Comparison of Trichoglossum species

\begin{tabular}{|c|c|c|c|c|c|}
\hline Species name & Fruiting body & Setae & Asci & Ascospores & References \\
\hline $\begin{array}{l}\text { Trichoglossum } \\
\text { cheliense F.L. } \\
\text { Tai }\end{array}$ & $\begin{array}{l}\text { Clavate, } 3-6 \mathrm{~cm} \\
\text { long, dark brown; } \\
\text { ascogenous portion } \\
\text { elliptic, compressed } \\
0.5-1.4 \mathrm{~cm} \text { long, } 5- \\
10 \mathrm{~mm} \text { wide; stipe } \\
3-4.5 \mathrm{~cm} \text { long, } 1.5- \\
3 \mathrm{~mm} \text { thick }\end{array}$ & Absent & $\begin{array}{l}\text { Clavate, } \\
200-231 \times \\
18-22 \mu \mathrm{m}, \\
8 \text {-spored }\end{array}$ & $\begin{array}{l}\text { Clavate- } \\
\text { cylindrical to } \\
\text { subcylindrical, } \\
\text { brown, } 89-151 \times \\
5-6 \mu \mathrm{m}, \text { mostly } \\
15-\text { septate, } \\
\text { rarely } 13-14- \\
\text { septate, }\end{array}$ & Tai, 1944 \\
\hline $\begin{array}{l}\text { Trichoglossum } \\
\text { confusum E.J. } \\
\text { Durand }\end{array}$ & $\begin{array}{l}\text { Black, clavate, } 1.5- \\
2.5 \mathrm{~cm} \text { long; } \\
\text { ascogenous portion } \\
\text { furrowed to } \\
\text { compressed; stipe } \\
1-1.5 \mathrm{~mm} \text { thick }\end{array}$ & $\begin{array}{l}\text { Abundant, } \\
\text { dark } \\
\text { brown, } \\
\text { acuminate }\end{array}$ & $\begin{array}{l}\text { Narrowly } \\
\text { clavate, } \\
150-200 \times \\
12-16 \mu \mathrm{m}, \\
8 \text {-spored }\end{array}$ & $\begin{array}{l}\text { Sub-cylindric, } \\
45-75 \times 5-6 \mu \mathrm{m}, \\
\text { mostly } 7 \text {-septate, } \\
\text { rarely 3-7- } \\
\text { septate }\end{array}$ & Durand, 1921 \\
\hline $\begin{array}{l}\text { Trichoglossum } \\
\text { farlowii } \\
\text { (Cooke) E.J. } \\
\text { Durand }\end{array}$ & $\begin{array}{l}\text { Scatered or } \\
\text { crowded, black or } \\
\text { brownish black, } \\
\text { clavate, } 3-8 \mathrm{~cm} \\
\text { long; ascogenous } \\
\text { portion compressed } \\
3-15 \mathrm{~mm} \text { wide; } \\
\text { stipe } 1.5-4 \mathrm{~mm} \\
\text { thick }\end{array}$ & $\begin{array}{l}\text { Abundant, } \\
\text { dark } \\
\text { brown }\end{array}$ & $\begin{array}{l}\text { Clavate, } \\
150-180 \times \\
15-20 \mu \mathrm{m}, \\
8 \text {-spored }\end{array}$ & $\begin{array}{l}\text { Sub-cylindric to } \\
\text { clavate-cylindric, } \\
45-90 \times 5-7 \mu \mathrm{m} \text {, } \\
\text { mostly } 3 \text {-septate, } \\
\text { rarely } 0-5 \text { or } \\
\text { more }\end{array}$ & Durand, 1908 \\
\hline $\begin{array}{l}\text { Trichoglossum } \\
\text { gracile Pat. }\end{array}$ & $\begin{array}{l}\text { Black, } 1.8-6 \mathrm{~cm} \\
\text { long; ascogenous } \\
\text { portion elliptic, } \\
\text { rounded, rarely } \\
\text { slightly } \\
\text { compressed, } 2-4 \\
\text { mm diam; stipe } \\
0.5-1 \mathrm{~mm} \text { thick }\end{array}$ & Present & $\begin{array}{l}\text { Clavate- } \\
\text { cylindrical, } \\
168-237 \times \\
16-19 \mu \mathrm{m}, \\
8 \text {-spored }\end{array}$ & $\begin{array}{l}\text { Clavate- } \\
\text { cylindrical, } \\
\text { brown, 110-162 } \\
\times 5-6 \mu \mathrm{m}, 15- \\
\text { septate }\end{array}$ & $\begin{array}{l}\text { Patouillard } \\
1909\end{array}$ \\
\hline $\begin{array}{l}\text { Trichoglossum } \\
\text { hirsutum } \\
\text { (Pers.) Boud. }\end{array}$ & $\begin{array}{l}\text { Scatered, } \\
\text { gregarious, black to } \\
\text { brownish black, } \\
\text { clavate to capitate, } \\
1-8 \mathrm{~cm} \text { long; } \\
\text { ascogenous portion } \\
\text { compressed upto } 2 \\
\text { cm long, } 2-5 \mathrm{~mm} \\
\text { wide; stipe } 2-3 \mathrm{~mm} \\
\text { thick }\end{array}$ & $\begin{array}{l}\text { Abundant, } \\
\text { dark } \\
\text { brown, } \\
\text { acuminate }\end{array}$ & $\begin{array}{l}\text { Clavate, } \\
180-275 \times \\
18-25 \mu \mathrm{m}, \\
8 \text {-spored }\end{array}$ & $\begin{array}{l}\text { Fusoid-clavate } \\
\text { cylindric, } 80-170 \\
\times 5-7 \mu \mathrm{m}, 15- \\
\text { septate }\end{array}$ & Imai, 1941 \\
\hline $\begin{array}{l}\text { Trichoglossum } \\
\text { kunmingense } \\
\text { F.L. Tai }\end{array}$ & $\begin{array}{l}1.5-3.5 \mathrm{~cm} \text { long; } \\
\text { ascogenous portion } \\
\text { lanceolate to } \\
\text { ellipticle, } \\
\text { compressed } 5.5-8 \\
\text { mm long, } 3-4 \mathrm{~mm} \\
\text { wide; stipe } 2-3 \mathrm{~mm} \\
\text { thick }\end{array}$ & Absent & $\begin{array}{l}\text { Clavate, } \\
175-225 \times \\
19-25 \mu \mathrm{m}, \\
8 \text {-spored }\end{array}$ & $\begin{array}{l}\text { Clavate } \\
\text { cylindrical to } \\
\text { sub-cylindrical, } \\
\text { brown, 104-144 } \\
\times 6-8 \mu \mathrm{m}, 7- \\
\text { septate, rarely 3- } \\
\text { 6-septate }\end{array}$ & Tai, 1944 \\
\hline
\end{tabular}


Table 1 continued, comparison of Trichoglossum species

\begin{tabular}{|c|c|c|c|c|c|}
\hline Species name & Fruiting body & Setae & Asci & Ascospores & References \\
\hline $\begin{array}{l}\text { Trichoglossum } \\
\text { octopartitum } \\
\text { Mains }\end{array}$ & $\begin{array}{l}\text { Clavate, black, } 1.5- \\
4 \mathrm{~cm} \text { long; } \\
\text { ascogenous portion } \\
2-4 \mathrm{~mm} \text { wide; stipe } \\
1-1.5 \mathrm{~mm} \text { thick }\end{array}$ & $\begin{array}{l}\text { Dark } \\
\text { brown, } \\
\text { acuminate }\end{array}$ & $\begin{array}{l}\text { Clavate, } \\
175-200 \times \\
18-20 \mu \mathrm{m}, \\
8 \text {-spored }\end{array}$ & $\begin{array}{l}\text { Fusoid to fusoid- } \\
\text { clavate, } 80-150 \\
\times 4-5.5 \mu \mathrm{m}, \\
\text { brown, } 7 \text {-septate, } \\
\text { rarely less }\end{array}$ & Mains, 1940 \\
\hline $\begin{array}{l}\text { Trichoglossum } \\
\text { persoonii F.L. } \\
\text { Tai }\end{array}$ & $\begin{array}{l}\text { Black, } 2.5-4 \mathrm{~cm} \\
\text { long; ascogenous } \\
\text { portion sub-globose } \\
\text { to ovate, rounded, } \\
5-9 \text { mm long, } 3-8 \\
\text { mm wide; stipe } 1-3 \\
\text { cm long, } 1-2 \mathrm{~mm} \\
\text { thick }\end{array}$ & Absent & $\begin{array}{l}\text { Clavate, } \\
225-275 \times \\
18-23 \mu \mathrm{m}, \\
8 \text {-spored }\end{array}$ & $\begin{array}{l}\text { Clavate } \\
\text { cylindrical, } \\
\text { brown, 162-200 } \\
\times 5-6 \mu \mathrm{m}, \\
\text { mostly } 15-19- \\
\text { septate, rarely } \\
\text { 13-20-septate }\end{array}$ & Tai, 1944 \\
\hline $\begin{array}{l}\text { Trichoglossum } \\
\text { peruvianum } \\
\text { E.K. Cash }\end{array}$ & $\begin{array}{l}\text { Narrow-clavate to } \\
\text { clavate-cylindrical, } \\
\text { fuscous black, 1- } \\
2.5 \mathrm{~cm} \\
\text { long;ascogenous } \\
\text { portion, } \\
\text { occasionally } \\
\text { compressed, } 1.5-2 \\
\text { mm in diameter; } \\
\text { stipe } 5-10 \times 1 \mathrm{~mm}\end{array}$ & $\begin{array}{l}\text { Dark } \\
\text { brown, } \\
\text { slightly } \\
\text { constricted } \\
\text { and paler } \\
\text { at the } \\
\text { base, acute } \\
\text { at the tips }\end{array}$ & $\begin{array}{l}\text { Long- } \\
\text { cylindrical, } \\
\text { narrowed } \\
\text { sharply at } \\
\text { the } \\
\text { apex and } \\
\text { gradually } \\
\text { toward the } \\
\text { base, } 120- \\
130 \times 8-11 \\
\mu \mathrm{m}, 8- \\
\text { spored, }\end{array}$ & $\begin{array}{l}\text { Elongate-fusoid, } \\
\text { olivaceous, } \\
50-75 \times 2.5-3.5 \\
\mu \mathrm{m}, \text { uniformly } 7 \text { - } \\
\text { septate }\end{array}$ & Cash, 1958 \\
\hline $\begin{array}{l}\text { Trichoglossum } \\
\text { qingchengense } \\
\text { W.Y. Zhuang }\end{array}$ & $\begin{array}{l}\text { Dark brown to } \\
\text { black, } 2-2.6 \mathrm{~cm} \\
\text { long; ascogenous } \\
\text { portion broadly } \\
\text { clavate, compressed } \\
7-9 \times 3-4 \mathrm{~mm} \text {; } \\
\text { stipe } 6-12 \times 1.5- \\
1.8 \mathrm{~mm}\end{array}$ & $\begin{array}{l}\text { Very few, } \\
\text { brown }\end{array}$ & $\begin{array}{l}\text { Clavate, } \\
190-230 \times \\
20-24 \mu \mathrm{m}, \\
8 \text {-spored, } \\
\text { sometimes } \\
\text { containing } \\
\text { fewer than } \\
8\end{array}$ & $\begin{array}{l}\text { Sub-cylindrical, } \\
\text { brown, } 76-117 \times \\
6.5-7.7 \mu \mathrm{m}, \\
\text { mostly } 7-9- \\
\text { septate, rarely 6- } \\
\text { septate }\end{array}$ & $\begin{array}{l}\text { Zhuang \& } \\
\text { Wang, } 1997\end{array}$ \\
\hline $\begin{array}{l}\text { Trichoglossum } \\
\text { rasum Pat. }\end{array}$ & $\begin{array}{l}\text { Brownish black to } \\
\text { black, clavate to } \\
\text { broadly spathulate, } \\
15 \text { cm long; } \\
\text { ascogenous portion } \\
\text { compressed, 6-20 } \\
\text { mm wide; stipe 2-3 } \\
\text { mm thick }\end{array}$ & Present & $\begin{array}{l}\text { Clavate, } \\
200 \times 20 \\
\mu \mathrm{m}, \\
\text { usually } 8- \\
\text { spored }\end{array}$ & $\begin{array}{l}\text { Mostly fusoid- } \\
\text { clavate, to } \\
\text { fusoid, } 115-140 \\
\times 7-9 \mu \mathrm{m}, \\
\text { mostly } 7-9 \text { - } \\
\text { septate }\end{array}$ & $\begin{array}{l}\text { Patouillard, } \\
1910\end{array}$ \\
\hline $\begin{array}{l}\text { Trichoglossum } \\
\text { rehmianum } \\
\text { (Henn.) E.J. } \\
\text { Durand }\end{array}$ & $\begin{array}{l}\text { Scatered to } \\
\text { crowded, black to } \\
\text { brownish black, } \\
\text { clavate, } 3-10 \mathrm{~cm} \\
\text { long; ascogenous } \\
\text { portion compressed } \\
4-10 \mathrm{~mm} \text { wide; } \\
\text { stipe } 2-6 \mathrm{~mm} \text { thick }\end{array}$ & $\begin{array}{l}\text { Abundant, } \\
\text { dark } \\
\text { brown, } \\
\text { acuminate }\end{array}$ & $\begin{array}{l}\text { Clavate, } \\
165-200 \times \\
15-18 \mu \mathrm{m}, \\
8 \text {-spored }\end{array}$ & $\begin{array}{l}\text { Sub-cylindric, } \\
\text { narrowing below, } \\
60-125 \times 5-6 \\
\mu \mathrm{m}, \text { light brown, } \\
\text { mostly } 7 \text {-septate }\end{array}$ & Durand, 1908 \\
\hline
\end{tabular}


Table 1 continued, comparison of Trichoglossum species

\begin{tabular}{|c|c|c|c|c|c|}
\hline Species name & Fruiting body & Setae & Asci & Ascospores & $\begin{array}{c}\text { Reference } \\
\text { S } \\
\end{array}$ \\
\hline $\begin{array}{l}\text { richoglossum } \\
\text { sinicum F.L. } \\
\text { Tai }\end{array}$ & $\begin{array}{l}\text { Clavate, black, } 5.5-7 \\
\text { cm long; ascogenous } \\
\text { portion compressed } \\
2.5-3 \mathrm{~cm} \text { long, } 0.8-1 \\
\text { cm thick; stipe } 3-4 \mathrm{~cm} \\
\text { long, } 3.5-5 \mathrm{~mm} \text { thick }\end{array}$ & Absent & $\begin{array}{l}\text { Clavate- } \\
\text { cylindrical, 237- } \\
281 \times 21-26 \mu \mathrm{m}, \\
\text { 8-spored, rarely } \\
\text { 4-spored }\end{array}$ & $\begin{array}{l}\text { Cylindrical- } \\
\text { clavate, brown, } \\
147-175 \times 6-7 \\
\mu \mathrm{m}, \text { mostly } 15- \\
\text { septate, rarely } \\
7-15 \text {-septate }\end{array}$ & Tai, 1944 \\
\hline $\begin{array}{l}\text { Trichoglossum } \\
\text { tetrasporum } \\
\text { Sinden \& } \\
\text { Fitzp. }\end{array}$ & $\begin{array}{l}\text { Black, clavate, } 3-8 \mathrm{~cm} \\
\text { long; ascogenous } \\
\text { portion compressed; } \\
\text { stipe } 1-2 \mathrm{~mm} \text { thick }\end{array}$ & $\begin{array}{l}\text { Dark } \\
\text { brown, } \\
\text { acuminate }\end{array}$ & $\begin{array}{l}\text { Clavate, } 175- \\
200 \times 20-25 \mu \mathrm{m}, \\
\text { 4-spored, } \\
\text { ocassinally with } \\
\text { fewer spores }\end{array}$ & $\begin{array}{l}\text { Clavate to } \\
\text { fusoid-clavate, } \\
110-150 \times 6-7 \\
\mu \mathrm{m}, \text { dark brown, } \\
\text { mostly } 15- \\
\text { septate, rarely } \\
0-17 \text {-septate }\end{array}$ & $\begin{array}{l}\text { Sinden, \& } \\
\text { Fitzpatric, } \\
1930\end{array}$ \\
\hline $\begin{array}{l}\text { Trichoglossum } \\
\text { variabile E.J. } \\
\text { Durand) } \\
\text { Nannf. }\end{array}$ & $\begin{array}{l}\text { Dark brown to black, } \\
\text { clavate, } 2-4 \mathrm{~cm} \text { long; } \\
\text { ascogenous portion } \\
\text { compressed; stipe } 1 \\
\text { mm thick }\end{array}$ & $\begin{array}{l}\text { Abundant, } \\
\text { dark brown, } \\
\text { acuminate }\end{array}$ & $\begin{array}{l}\text { Clavate, } 150- \\
200 \times 18-20 \mu \mathrm{m}, \\
8 \text {-spored }\end{array}$ & $\begin{array}{l}\text { Sub-fusoid or } \\
\text { fusoid-clavate, } \\
80-150 \times 4.5-6 \\
\mu \mathrm{m}, \text { mostly } 10- \\
13 \text {-septate, } \\
\text { rarely 4-16- } \\
\text { septate }\end{array}$ & $\begin{array}{l}\text { Nannfeldt, } \\
1942\end{array}$ \\
\hline $\begin{array}{l}\text { Trichoglossum } \\
\text { velutipes } \\
\text { (Peck) E.J. } \\
\text { Durand }\end{array}$ & $\begin{array}{l}\text { Scatered to crowded, } \\
\text { black to brownish } \\
\text { black, clavate, } 2-5 \mathrm{~cm} \\
\text { long; ascogenous } \\
\text { portion compressed } 2- \\
12 \mathrm{~mm} \text { wide; stipe } \\
1.5-2.5 \mathrm{~mm} \text { thick }\end{array}$ & $\begin{array}{l}\text { Abundant, } \\
\text { dark brown, } \\
\text { acuminate }\end{array}$ & $\begin{array}{l}\text { Clavate, } 180- \\
200 \times 16-20 \mu \mathrm{m}, \\
\text { 4-spored }\end{array}$ & $\begin{array}{l}\text { Clavate, } 90-160 \\
\times 6-7 \mu \mathrm{m}, \\
\text { mostly } 9- \\
\text { septate, rarely } \\
0-13 \text {-septate }\end{array}$ & $\begin{array}{l}\text { Durand, } \\
1908\end{array}$ \\
\hline $\begin{array}{l}\text { Trichoglossum } \\
\text { walteri (Berk.) } \\
\text { E.J. Durand }\end{array}$ & $\begin{array}{l}\text { Up to } 34 \mathrm{~mm} \text { high; } \\
\text { Clavula about } 10 \times 2.5 \\
\text { mm, ligulate, with } \\
\text { median } \\
\text { groove } \\
\text { and obtuse } \\
\text { apex, densely setose, } \\
\text { dull, black-brown; } \\
\text { Stipe } 24 \times 1 \mathrm{~mm}\end{array}$ & $\begin{array}{l}\text { Black to } \\
\text { brown, } \\
\text { thick walled }\end{array}$ & $\begin{array}{l}120-217 \times 18- \\
24 \mu \mathrm{m}, 8 \text {-spored. }\end{array}$ & $\begin{array}{l}\text { Cylindrical- } \\
\text { clavate } \\
\text { to acicuiar, 49- } \\
108 \times 5-8 \mu \mathrm{m}, \\
0-7 \text {-septate, } \\
\text { brown }\end{array}$ & Imai, 1941 \\
\hline $\begin{array}{l}\text { Trichoglossum } \\
\text { wrightii (E.J. } \\
\text { Durand) E.J. } \\
\text { Durand }\end{array}$ & $\begin{array}{l}\text { Black, velvety, } \\
\text { clavate, variable in } \\
\text { size; ascogenous } \\
\text { portion irregular }\end{array}$ & $\begin{array}{l}\text { Cystidia } \\
\text { present }\end{array}$ & $\begin{array}{l}\text { Clavate, } \\
\text { cylindric, } 250- \\
265 \times 20-25 \mu \mathrm{m}, \\
8 \text {-spored }\end{array}$ & $\begin{array}{l}\text { Clavate, brown, } \\
105-145 \times 7 \\
\mu \mathrm{m}, \text { mostly } 8-9- \\
\text { septate, rarely } \\
5-6 \text { or } 7 \text {-septate }\end{array}$ & $\begin{array}{l}\text { Durand, } \\
1921\end{array}$ \\
\hline $\begin{array}{l}\text { Trichoglossum } \\
\text { yunnanense } \\
\text { F.L. Tai }\end{array}$ & $\begin{array}{l}\text { Black, } 3.5-7.5 \mathrm{~cm} \\
\text { long; ascogenous } \\
\text { portion ellipticle to } \\
\text { subglobose, } \\
\text { compressed; stipe } 4.5- \\
7 \mathrm{~cm} \text { long, } 2-3 \mathrm{~mm} \\
\text { thick }\end{array}$ & Absent & $\begin{array}{l}\text { Cylindrical- } \\
\text { clavate, 237-294 } \\
\times 19-22 \mu \mathrm{m}, 4- \\
\text { spored, rarely 2- } \\
8 \text {-spored }\end{array}$ & $\begin{array}{l}\text { Clavate } \\
\text { cylindrical, } \\
\text { brown, 143-187 } \\
\times 6-7 \mu \mathrm{m}, 15- \\
\text { septate, rarely } \\
\text { 16-septate }\end{array}$ & Tai, 1944 \\
\hline
\end{tabular}



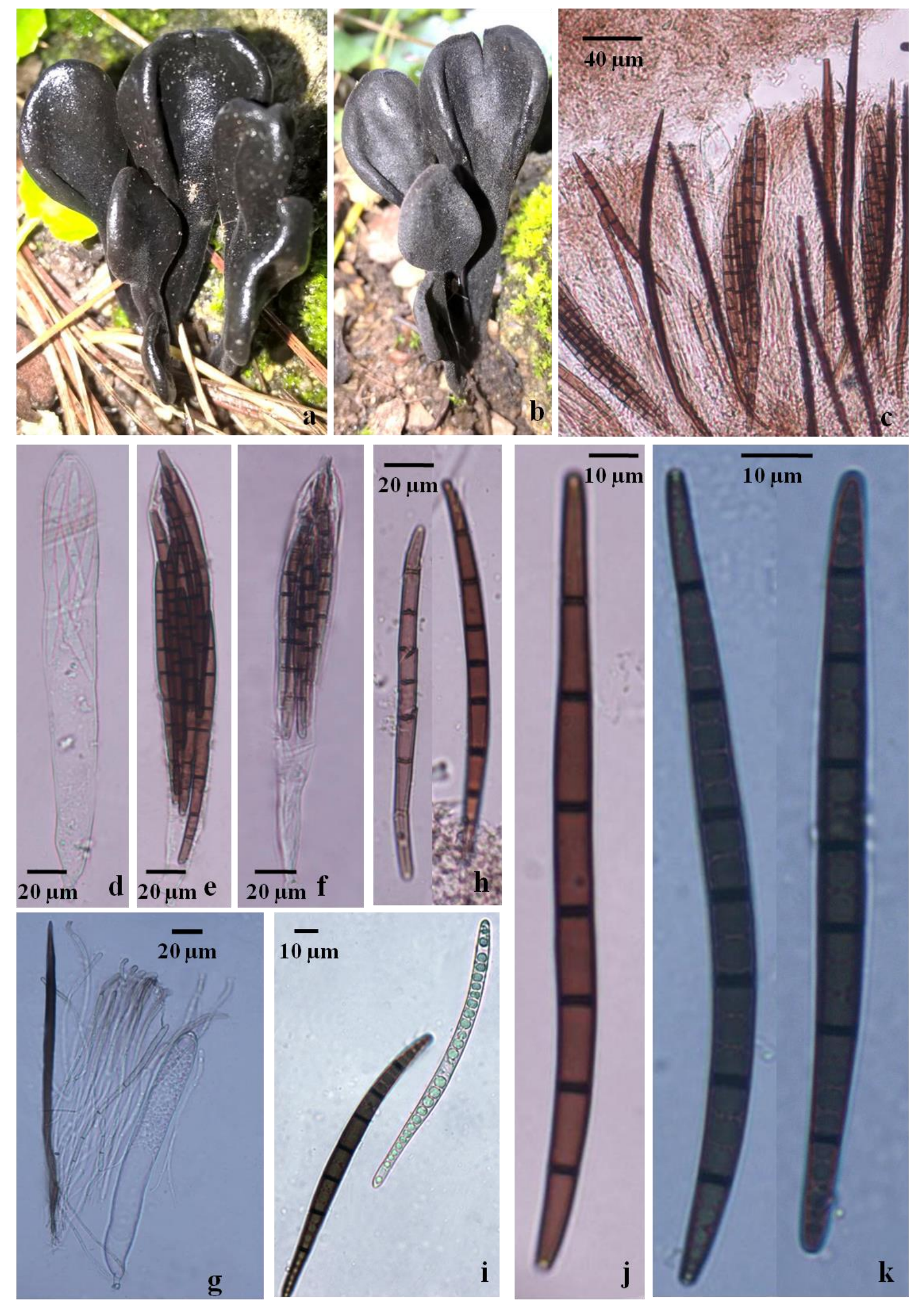

Figure 2 - Trichoglossum rasum. a, b Habit, c Asci with setae, d Young ascus with ascospores, e, $\mathrm{f}$ Asci with ascospores, $\mathrm{g}$ Young ascus with setae and paraphyses, $\mathrm{h}-\mathrm{k}$ Ascospores 
Table 2 Sequence data used in combined ITS and LSU analyses. Newly deposited sequences are in bold

\begin{tabular}{cccc}
\hline Taxon & Acession no. & ITS & LSU \\
\hline Geoglossum simile & SAV 10136 & KF854293 & KF854301 \\
Geoglossum simile & T. Galante 00009 & KF944382 & KF944384 \\
Geoglossum simile & SAV 10129 & KF854286 & KF854294 \\
Geoglossum dunense & TUR-A 199830 & KP744516 & KP744517 \\
Geoglossum umbratile & K(M) 169625 & KC222127 & KC222140 \\
Nothomitra cinnamomea & ILLS:61042 & JQ256424 & JQ256439 \\
Glutinoglossum heptaseptatum & ILLS 63754 & KC222130 & KC222143 \\
Glutinoglossum glutinosum & ILLS 67352 & KC222128 & KC222141 \\
Glutinoglossum methvenii & PDD:103604 & KP690097 & KP690109 \\
Glutinoglossum australasicum & PDD:103623 & KP690088 & KP690100 \\
Hemileucoglossum littorale & C:35673 & KP657561 & KP657566 \\
Hemileucoglossum alveolatum & MICH s.n. & KP657560 & KP657565 \\
Trichoglossum hirsutum & HKAS 55133 & KC222133 & KC222146 \\
Trichoglossum hirsutum & ILLS 67355 & KC222132 & KC222145 \\
Trichoglossum octopartitum & ILLS:61046 & JQ256429 & JQ256443 \\
Trichoglossum hirsutum & ILLS:61045 & JQ256428 & JQ256442 \\
Trichoglossum hirsutum & OSC 100017 & NR_121205 & NG_042405 \\
Trichoglossum variabile & ERRO 2011012206 & KP144106 & - \\
Trichoglossum variabile & ERRO 2009111802 & KP144105 & - \\
Trichoglossum rasum & HCIO 52051 & KY457226 & KY457227 \\
Dothidea sambuci & CBS 198.58 & AY930109 & AF382387 \\
\hline
\end{tabular}

\section{References}

Agnihothrudu V, Barua GCS. 1962 - Trichoglossum hirsutum (Pers.) Boud. Var longisporum (Tai) E. B. Mains from assam with a note on Indian geoglossaceae. Current science 9, 386-387.

Boudier JLE. 1885 - Nouvelle classification naturelle des Discomycetese charnusconnus generalement sous le nom de Pezizales. Bulletin de la Société. Mycologique de France 1, 91-120.

Boudier JLE. 1907. Histoire et classification des discomycètes d'Europe. Paul Klincksieck, Paris 1221

Cash EK. 1958 - Some new or rare Discomycetes from South America. J. Wash. Acad. Sci. 48(8), 256-259.

Crous PW, Braun U, Wingfield MJ, Wood AR et al. 2009 - Phylogeny and taxonomy of obscure genera of microfungi. Persoonia 22, 139-161. http://dx.doi: 10.3767/003158509X461701

Das K. 2009 - Mushrooms of Sikkim I: Barsey Rhododendron Sanctuary. Sikkim State Biodiversity Board, Gangtok and Botanical Survey of India, Kolkata. 1-160.

Drummond AJ, Ashton B, Buxton S, Cheung M, Cooper A et al. 2010 - Geneious v. 5.1, available from http://www.geneious.com.

Durand EJ. 1908 - The Geoglossaceae of North America. In Annls mycol. 6, 387-477

Durand EJ. 1921 - New or noteworthy Geoglossaceae. Mycologia 13, 184-187.

Hustad VP, Miller AN, Dentinger BTM, Cannon PF. 2013 - Generic circumscriptions in Geoglossomycetes. Persoonia 31, 101-111.

Imai S. 1941 - Geoglossaceae Japoniae. In J. Fac. Agric. Hokkaido imp. Univ. 45, 155-264.

Index Fungorum 2017 - www.indexfungorum.org/names/Names.asp

Jayasiri SC, Hyde KD, Ariyawansa HA, Bhat DJ et al. 2015 - The Faces of Fungi database: Fungal names linked with morphology, phylogeny and human impacts. Fungal Diversity 74, 3-18.

Kumar S, Nei M, Dudley J, Tamura K. 2008 - MEGA: a biologist-centric software for evolutionary analysis of DNA and protein sequences. Briefings in Bioinformatics 9, 299-306.

Mains EB. 1940 - New and unusual species of the Geoglossaceae. Amer. Journ. of Bot. 27: 322326. 
Nannfeldt JA. 1942 - The Geoglossaceae of Sweden. (With regard also to the surrounding countries.) In Ark. Bot. 1-67.

Patouillard N. 1909 - Champignons de la Nouvelle Caledonie (suite). In Bull. Soc. mycol. France 35,129-134.

Prabhugaonkar A, Bhat DJ. 2011 - New record of Megacapitula villosa and Paradictyoarthrinium diffractum from India. Mycosphere 2(4), 463-467.

Report: State of the Environment Report, Meghalaya. 2005. Department of Environment and Forests, Government of Meghalaya, 1-76.

Rifai MA. 1965 - Discomycete flora of Asia, Precursor III: Observations on Javanese species of Trichoglossum. Lloydia 28, 113-119.

Sinden JW, Fitzpatrick HM. 1930 - A new Trichoglossum. Mycol. 22, 55-60.

Tai FL. 1944 - Studies in the Geoglossaceae of Yunnan. Lloydia 7(2), 146-162.

Teng SC. 1934 - Notes on Discomycetes from China. Sinensia 5, 431-465.

Thind KS, Singh, H. 1965 - The Helotiales of India-III. Ind. bot. Soc. 43, 529-542.

White TJ, Bruns T, Lee S, Taylor J. 1990 - Amplification and direct sequencing of fungal ribosomal RNA genes for phylogenetics. In: Innis MA, Gelfand DH, Sninsky JJ, White TJ, (Eds.) PCR protocols: a guide to methods and applications. Academic Press, New York, USA, 315-322.

Zhuang WY, Wang Z. 1997 - Some new species and new records of discomycetes in China VII Mycotaxon 63, 307-321. 